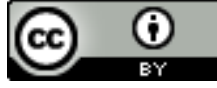

\title{
POLITICA SALARIAL DE LAS UNIVERSIDADES PÚBLICAS: MÉXICO Y VENEZUELA
}

\author{
SALARY POLICY OF PUBLIC UNIVERSITIES: MEXICO AND VENEZUELA \\ POLÍTICA SALARIAL DAS UNIVERSIDADES PÚBLICAS: MÉXICO E VENEZUELA
}

Luis E. Torres-Núñez i

\begin{abstract}
RESUMEN: La formación de profesionales de calidad y la generación de tecnología y ciencia pertinente son al menos dos elementos fundamentales para el desarrollo de los países latinoamericanos y, tal situación se encuentra estrechamente relacionada al impacto que se genere como resultado del trabajo académico mayormente desarrollado en las universidades de la región. El presente trabajo procura caracterizar la política salarial de los académicos mexicanos y venezolanos en el periodo del año 2000 al 2010. Es una investigación comparativa y de carácter documental. El ensayo se estructura en dos dimensiones a saber: la presentación de los datos que contienen los elementos significativos de los sistemas de educación superior (matricula, instituciones, política de financiamiento y los académicos); en segundo lugar, se expone la estructura de la política salarial, se estudian las realidades y se precisan algunas diferencias sustantivas. Finalmente el estudio comparativo revela el incumplimiento de ambos Estados en relación a las definiciones salariales contempladas en sus textos constitucionales. La política mexicana de salarios académicos "estructura de incentivos" de tipo heterologados enseñan beneficios económicos de carácter inmediato para la sobrevivencia del académico y no le garantizan una pensión digna para el disfrute de su vejez; mientras que la política venezolana de salarios de tipo homologados precisa el incumplimiento legal de las "Normas de Homologación" por parte del Estado Venezolano desde hace más de tres décadas para el incremento salarial de los académicos.
\end{abstract}

PALABRAS-CLAVES: Política salarial. Educación superior. Políticas públicas.

ABSTRACT: The training of quality professionals and the generation of relevant technology and science are at least two fundamental elements for the development of Latin American countries and, this situation is closely related to the impact that is generated as a result of the academic work mostly developed in the universities of the region. This paper search to characterize the wage policy of the academics Mexican and Venezuelan in the period from 2000 to 2010. It is a comparative and documentary research. The essay is structured in two dimensions namely: The presentation of data containing the significant elements of higher education systems (enrollment, institutions, funding policy and academics); secondly, it exposed the structure of the wage policy, it study the realities and detail some substantive differences. Finally the comparative study reveals the noncompliance of the two States in relation to the wage definitions contemplated in their constitutional texts. The policy Mexican of heterologous "incentive structure" type academic teaches immediate economic benefits for the academic's survival and does not guarantee him a decent pension for the enjoyment of his old age; while the Venezuelan policy of wages of type homologated specifies the legal non-compliance of the "Homologation standards" by the Venezuelan State for more than three decades for the salary increase of the academics.

KEYWORDS: Salary policy. Higher education. Public policies.

RESUMO: O Formação de profissionais de qualidade e a geração de tecnologia e ciência relevantes são pelo menos dois elementos fundamentais para o desenvolvimento de países da América Latina e, esta situação está intimamente relacionada ao impacto gerado como resultado do trabalho acadêmico desenvolvido principalmente nas universidades da região. Este artigo procura caracterizar a política salarial dos acadêmicos mexicanos e venezuelanos no período de 2000 a 2010. É uma pesquisa comparativa e documental. O ensaio está estruturado em duas dimensões, a saber: a apresentação dos dados contendo os elementos significativos dos sistemas de

Submetido em: 14/03/2017 - Aceito em: 08/06/2017 - Publicado em: 14/07/2017.

n.2

p.379-400


ensino superior (matrícula, instituições, política de financiamento e acadêmicos); em segundo lugar, expôs a estrutura da política salarial, estuda as realidades e detalha algumas diferenças substantivas. Finalmente, o estudo comparativo revela a não cumprimento de ambos os Estados em relação às definições salariais contempladas em seus textos constitucionais. A política do salario acadêmico mexicana de tipos heterólogos de "estrutura de incentivo" ensina benefícios econômicos imediatos para a sobrevivência do acadêmico e não lhe garante uma pensão decente para o gozo da sua velhice; Enquanto a política venezuelana de salários acadêmicos de tipo homologada amostra o incumprimento legal dos "Padrões de Homologação" pelo Estado venezuelano por mais de três décadas para o aumento salarial dos acadêmicos.

PALABRAS CLAVES: Política salarial. Educação superior. Políticas públicas.

\section{INTRODUCCIÓN}

México y Venezuela pertenecen a la región latinoamericana, la cual históricamente han presenciado elementos que le son comunes: un solo idioma, una religión predominantemente cristiana (con mayor énfasis del catolicismo) y hasta una visión común frente al mundo occidental. Pero, al igual que el resto de los países de la región, también han experimentan en diferentes medidas serios obstáculos para el desarrollo científico y tecnológico que le permitan mayor eficiencia de sus sistemas productivos, causa principal que le impiden superar la pobreza y los altos niveles de desigualdad social.

En este contexto son las universidades un actor fundamental para el desarrollo, la cual deberá en conjunto con el Estado definir, implantar y ejecutar acciones concretas que le permitan superar la situación antes descrita. De la misma forma, la Organización de Estados Iberoamericanos para la educación, la ciencia y la tecnología (OEI) señala: "el desarrollo de las naciones depende ahora más que nunca de la calidad de la formación a la que se accede en las universidades y del conocimiento que se pueda generar y acumular en ellas." (OEI, 2011, p. 137).

Ahora bien, la calidad de la formación que impartan las universidades de la región está estrechamente vinculada con el desarrollo efectivo de la profesión académica y esta a su vez, se encuentra influenciada por la política salarial que determinen los Estados en relación a los profesores universitarios. El presente estudio, tiene como propósito comparar la política salarial de los académicos mexicanos y venezolanos, toda vez que se procura comprender como esto influye en la gestión académica de las universidades. Para ello el trabajo se estructura en dos dimensiones a saber: la presentación de los datos que contienen los elementos significativos de los sistemas de educación superior (matricula, instituciones, política de financiamiento y los académicos); en segundo lugar, se expone la estructura de la política salarial, se estudian las realidades y se precisan algunas diferencias sustantivas.

V.3

n.2




\section{PRINCIPALES ELEMENTOS CARACTERÍSTICOS DE LOS SISTEMAS DE EDUCACIÓN SUPERIOR: MÉXICO Y VENEZUELA}

Es necesario iniciar el estudio identificando algunos elementos característicos de los sistemas educativos (SE) que configuran los "cambios en las dinámicas del crecimiento" de la realidad presente en la universidad mexicana y venezolana, estos cambios en su mayoría se corresponden con el crecimiento exponencial de los números en: matricula estudiantil; instituciones de educación superior (IES) y financiamiento universitario, que singularmente están condicionando en alguna medida, la realidad de los profesores universitarios. Para ello, procedemos a revisar los elementos antes señalados, para cada uno de los sistemas nacionales de educación superior (ES), entre los años 2000-2010:

\section{Población escolarizada en educación superior}

Si bien es cierto que la región ha experimentado incrementos exponenciales en materia de masificación de la educación superior, aún existen elementos controversiales como el acceso y participación de la sociedad. Este segmento, procura revisar de forma general el nivel de acceso y participación de la población mexicana y venezolana en materia de ES, con base a los datos señalados en el tabla número 1 "Evolución anual de la población nacional escolarizada en el pregrado"; allí se identifican para los años (2000-2010) la matrícula nacional de pregrado (discriminada en pública y privada) de ambos países, además se acompaña de la tasa de crecimiento (t-Crecimiento), la cual muestra cómo ha sido la evolución del crecimiento global de la matrícula en dicho periodo. Estos datos, se consideran necesarios para fundamentar el análisis toda vez que se consideran insuficientes los datos brutos de la matrícula. 


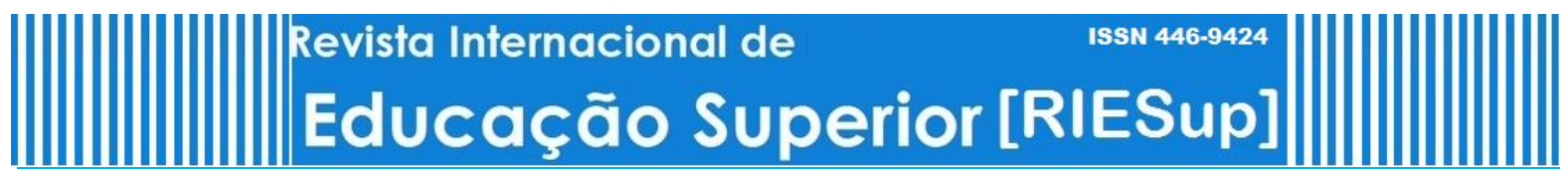

Artigo

DOI: $10.22348 /$ riesup.v3i2.7731

Tabla 1: Evolución anual de la población nacional escolarizada en el pregrado

\begin{tabular}{lcrcrrrrr}
\hline \multicolumn{1}{c}{ México $^{1}$} & \multicolumn{5}{c}{ Venezuela $^{2}$} \\
\hline & Pública & Privada* $^{2}$ Total & \multicolumn{1}{c}{ t-Crecimto } & Pública & Privada & Total & t-Crecimto \\
\hline 2000 & 1390241 & 657816 & 2050057 & 1,00 & 526270 & 368148 & 894418 & 1,00 \\
2001 & 1442666 & 704409 & 2149076 & 1,05 & 569695 & 401341 & 971036 & 1,09 \\
2002 & 1494564 & 742227 & 2238793 & 1,09 & 565037 & 448969 & 1014006 & 1,13 \\
2003 & 1556885 & 765896 & 2324784 & 1,13 & 626244 & 430634 & 1056878 & 1,18 \\
2004 & 1604142 & 780716 & 2386862 & 1,16 & 721821 & 448405 & 1170226 & 1,31 \\
2005 & 1647213 & 799513 & 2448731 & 1,19 & 917821 & 500482 & 1418303 & 1,59 \\
2006 & 1694934 & 833730 & 2530670 & 1,23 & 1270335 & 543635 & 1813970 & 2,03 \\
2007 & 1749184 & 874183 & 2625374 & 1,28 & 1486904 & 528236 & 2015140 & 2,25 \\
2008 & 1809921 & 895545 & 2707474 & 1,32 & 1518860 & 590471 & 2109331 & 2,36 \\
2009 & 1938921 & 908455 & 2849385 & 1,39 & 0 & 0 & 0 & 0 \\
2010 & 1996290 & 939363 & 2937663 & 1,43 & 1764048 & 590471 & 2354519 & 2.32 \\
Total tasa de crecimiento promedio & 1,21 & & & & 1,63
\end{tabular}

${ }^{1}$ Fuente: Gil Antón, M y Pérez García, M. (2006) Proyecto informe de la educación superior Iberoamericana: México. México DF. Y; Gil Antón, M y Pérez García, M. (2011) Proyecto informe de la educación superior Iberoamericana: México. México DF.

${ }^{2}$ Fuente: Memoria y cuenta del Ministerio para el poder popular para la educación universitaria, año 2008 y 2010.

T Crecimto= Tasa de Crecimiento; tomando el año 2000 como base.

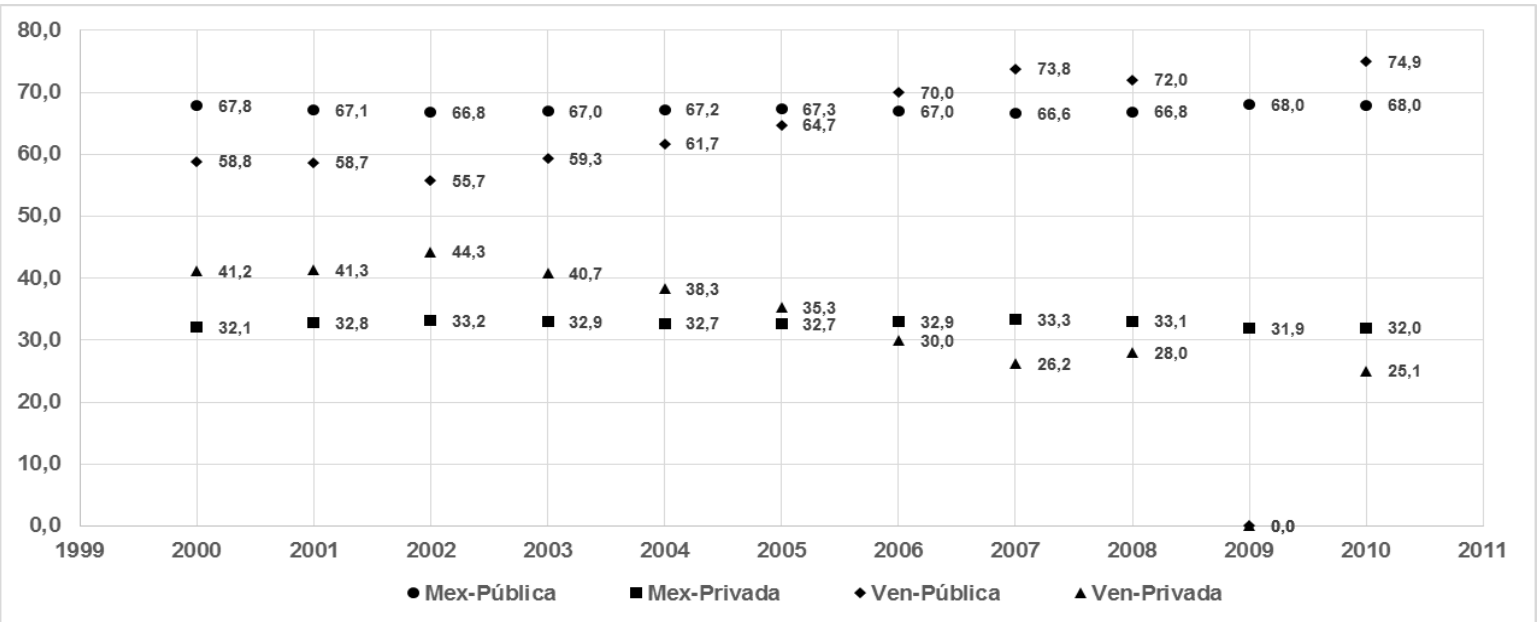

Grafico 1: Relación general de la proporción de la matricula pública y privada.

Para el caso mexicano, se identificó una tasa de crecimiento (t-Crecimiento) promedio general del 1,21 en el tiempo estudiado. En términos de tipo de la matrícula, se observan en la gráfica 1 "Relación general de la proporción de la matricula pública y privada" con muy leves variaciones porcentuales (incluso menores a un digito) entre los proveedores educativos (públicos-privados) durante todo el periodo de estudio, en promedio total se estimó una matrícula de $67,24 \%$ para el sector público y para el sector privado un promedio total de la matrícula del 32,68\%. Esta información, demuestra la estrategia de "continuidad, 
profundización y consolidación de las mismas políticas públicas en materia de ES en México", la cual ha mantenido el sistema inerte y con pocas variaciones por más de tres décadas. (Gil M. 2014).

Para el caso venezolano ${ }^{2}$, se observa una tasa de crecimiento (t-Crecimiento) promedio del 1,63 durante el periodo de estudio. La relación entre los proveedores de la educación superior en el sistema venezolano, estuvo caracterizada por una reconfiguración importante en el periodo de estudio, particularmente la matricula pública se incrementó en 16,08\%, al pasar de $58.84 \%$ en el año 2000 a $74.92 \%$ para el año 2010. Lo cual, causó un retroceso de la matricula privada de $41.16 \%$ inicialmente a $25.08 \%$ para el año 2010 . A diferencia de México, esta información evidencia la implementación de una redefinición de las políticas públicas en materia de educación superior en Venezuela -hacia la universalización de la educación universitaria- por parte del nuevo proyecto político del gobierno que se inicia desde 1999; para ello fue fundamental la aplicación de dos grandes políticas públicas: la creación de la Universidad Bolivariana de Venezuela y, la creación del programa social "Misión Sucre", (PARRA-SANDOVAL, 2011).

\section{Tejido institucional de los sistemas nacionales de educación superior}

La masificación de la educación superior en la región latinoamericana, sin lugar a dudas ha impactado el tejido institucional (públicos-privados) para que los Estados y el sector privado en su conjunto estén dando respuesta a las demandas sociales. Ahora bien, es necesario conocer el comportamiento de esta variable en el periodo 2000-2010, así podremos determinar en qué tipo de IES se abrieron las oportunidades de estudio y con ello comprender el tipo de desarrollo académico y las posibles tendencias de permanencia y contrataciones de las cuales están siendo objeto los profesores universitarios.

En este apartado, se identificará el número de las IES; además de conocer cómo se clasificaban (universitarias y no universitarias). Con ello, se podrá comprender cómo el tejido institucional y sus características de gestión académica, gobierno, control y financiamiento universitario están impactando sobre el comportamiento de los profesores universitarios. Para facilitar la caracterización de los países objeto del estudio, se muestran los datos para el caso mexicano en la tabla número 2 "Evolución del número de instituciones de educación superior

\footnotetext{
${ }^{2}$ Existe gran dificultad para la obtención de datos públicos (suficientes, oportunos y definitivos) en materia de educación superior en Venezuela. Se encontraron diferencias entre las cifras relativas a la matricula en varios documentos oficiales (Memorias y Cuenta del Ministerio del Poder Popular para la Educación Universitaria). Los datos fueron tomados, de los últimos documentos oficiales relativos al periodo de investigación; nos referimos en sentido particular a dos Memorias y Cuentas de los años 2008 y 2010 del Ministerio para el Poder Popular de Educación Universitaria. Asimismo señalamos la inexistencia de información acerca de la matrícula para el año 2009, razón por la cual fue omitida en la presente investigación.
} 
en México" y; en el caso venezolano se presentan los datos en la tabla número 3: "Evolución del número de instituciones de educación superior en Venezuela".

Tabla 2: Evolución del número de instituciones de educación superior en México.

\begin{tabular}{rrrrrrrrrrr}
\hline & Pública & \multicolumn{1}{c}{$\%$} & \multicolumn{1}{c}{ Privada } & \multicolumn{1}{c}{$\%$} & Total & Universitaria & $\%$ & $\begin{array}{c}\text { No- } \\
\text { Universitaria }\end{array}$ & $\%$ & Total \\
\hline 2000 & 504 & 35,59 & 912 & 64,41 & 1416 & 1371 & 96,82 & 45,00 & 3,18 & 1416,00 \\
2001 & 529 & 35,15 & 976 & 64,85 & 1505 & 1456 & 96,74 & 49,00 & 3,26 & 1505,00 \\
2002 & 570 & 34,28 & 1093 & 65,72 & 1663 & 1534 & 92,24 & 129,00 & 7,76 & 1663,00 \\
2003 & 581 & 32,90 & 1185 & 67,10 & 1766 & 1583 & 89,64 & 183,00 & 10,36 & 1766,00 \\
2004 & 615 & 34,67 & 1159 & 65,33 & 1774 & 1629 & 91,83 & 145,00 & 8,17 & 1774,00 \\
2005 & 818 & 36,11 & 1447 & 63,89 & 2265 & 2133 & 94,17 & 132,00 & 5,83 & 2265,00 \\
2006 & 839 & 35,05 & 1555 & 64,95 & 2394 & 2370 & 99,00 & 24,00 & 1,00 & 2394,00 \\
2007 & 856 & 34,53 & 1623 & 65,47 & 2479 & 2453 & 98,95 & 26,00 & 1,05 & 2479,00 \\
2008 & 872 & 33,89 & 1701 & 66,11 & 2573 & 2571 & 99,92 & 2,00 & 0,08 & 2573,00 \\
2009 & s.d & s.d & s.d & s.d & s.d & s.d & s.d & s.d & s.d & s.d \\
\hline
\end{tabular}

\section{Fuente:}

Gil Antón, M y Pérez García, M. (2006) Proyecto informe de la educación superior Iberoamericana: México.

México DF. Y; Gil Antón, M y Pérez García, M. (2011) Proyecto informe de la educación superior Iberoamericana: México. México DF.

s.d: sin datos; los estudios antes señalados, muestran la información para este periodo en términos de escuela, situación que altera las cifras y dificulta la relación IES-Escuela, en función de depurar los datos.

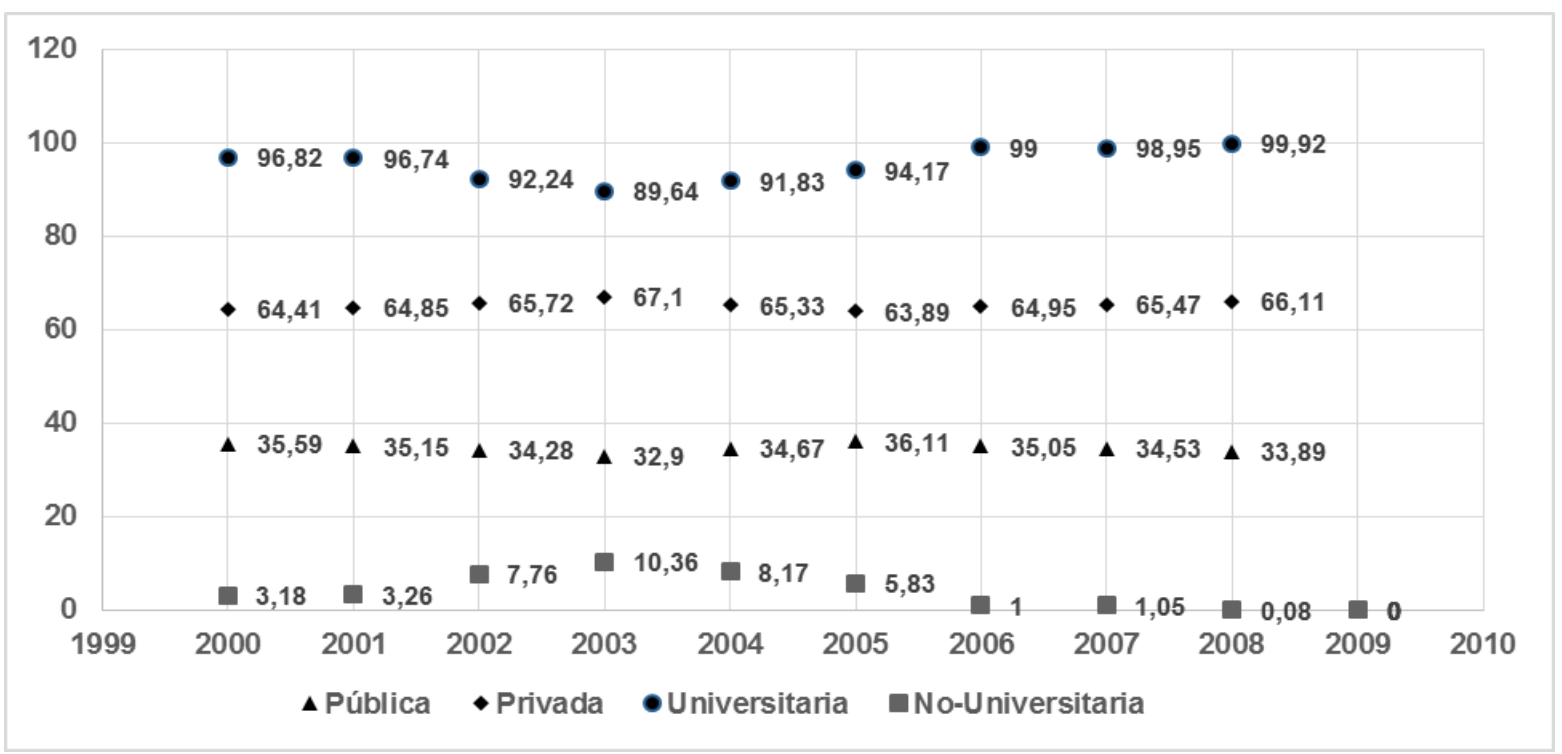

Grafico 2: Relación entre las IES pública vs privada; y su clasificación en universitaria y nouniversitaria en México. 
El tejido institucional mexicano alcanzó para el año 2008 la cantidad de 2573 IES; se evidencia como logró casi duplicarse en 1157 nuevas IES en el periodo objeto del estudio. La mayoría de las nuevas instituciones se corresponden al sector privado, el cual se ubica con un crecimiento porcentual del 55,57\%, es decir 789 nuevas IES privadas. Las IES se clasifican casi en totalidad por ser de tipo universitaria, para ello la Secretaría de Educación Pública las tipifica en dos rubros. En primer lugar, las instituciones de tipo universitaria, las cuales alcanzan 9 figuras jurídicas distintas a saber: universidades públicas federales, universidades públicas estatales, universidades públicas estatales con apoyo solidario, universidades tecnológicas, universidades politécnicas, universidades interculturales, institutos tecnológicos, centros públicos de investigación, educación normal superior; este sector representa casi la totalidad de la IES mexicanas ubicadas porcentualmente en un $96.82 \%$ para el año base (2000) y al culminar el periodo de estudio se incrementó al 99,92\% de la totalidad de las IES. En segundo lugar como: otras instituciones públicas lograron casi una desaparición del SE mexicano, al reducirse de 45 IES $(3,18 \%)$ en el año 2000 a dos 2 $(0,008 \%)$ IES-No universitarias para el año 2008.

La creciente creación de nuevas IES, obedece entre otras razones a la falta de coordinación de los instrumentos normativos y a la ausencia de criterios homogéneos entre los diferentes niveles del gobierno mexicano, situación que ha propiciado un fuerte incremento de las universidades privadas denominadas como de "absorción de la demanda residual" y con ello el cuestionamiento de la Secretaria de Educación Federal Pública para el reconcomiendo oficial. (GIL ANTÓN, 2011).

Tabla 3: Evolución del número de instituciones de educación superior en Venezuela.

\begin{tabular}{|c|c|c|c|c|c|c|c|c|c|c|}
\hline & Pública & $\%$ & Privada & $\%$ & Total & Universitaria & $\%$ & No-Universitaria & $\%$ & Total \\
\hline 2000 & 68 & 46,58 & 78 & 53,42 & 146 & 42 & 28,77 & 104 & 71,23 & 146 \\
\hline 2001 & 71 & 47,33 & 79 & 52,67 & 150 & 42 & 28,00 & 108 & 72,00 & 150 \\
\hline 2002 & 72 & 45,57 & 86 & 54,43 & 158 & 43 & 27,22 & 115 & 72,78 & 158 \\
\hline 2003 & 73 & 44,79 & 90 & 55,21 & 163 & 46 & 28,22 & 117 & 71,78 & 163 \\
\hline 2004 & 73 & 43,45 & 95 & 56,55 & 168 & 48 & 28,57 & 120 & 71,43 & 168 \\
\hline 2005 & 73 & 43,20 & 96 & 56,80 & 169 & 49 & 28,99 & 120 & 71,01 & 169 \\
\hline 2006 & 61 & 40,13 & 91 & 59,87 & 152 & 46 & 30,26 & 106 & 69,74 & 152 \\
\hline 2007 & 62 & 40,00 & 93 & 60,00 & 155 & 47 & 30,32 & 108 & 69,68 & 155 \\
\hline 2008 & 66 & 41,51 & 93 & 58,49 & 159 & 49 & 30,82 & 110 & 69,18 & 159 \\
\hline 2009 & 69 & 42,07 & 95 & 57,93 & 164 & 52 & 31,71 & 112 & 68,29 & 164 \\
\hline 2010 & 75 & 44,12 & 95 & 55,88 & 170 & 58 & 34,12 & 112 & 65,88 & 170 \\
\hline
\end{tabular}

\section{Fuente:}

García Guadilla, C. (2006) Informe de Venezuela. Proyecto CINDA: Educación superior en Iberoamérica 2006. Caracas

Parra Sandoval, M. (2011) La educación superior en Iberoamérica 2011. La educación superior en Venezuela 2005-2009. Maracaibo 


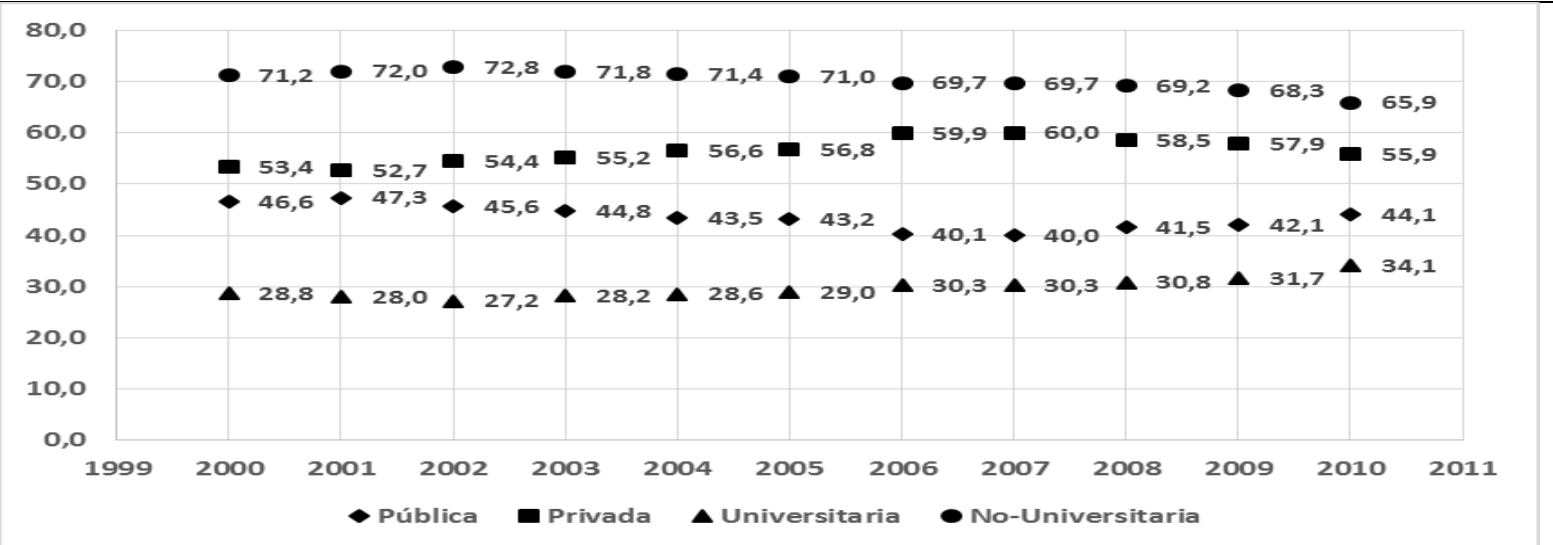

Grafico 2: Relación entre las IES pública vs privada; y su clasificación en universitaria y nouniversitaria en Venezuela.

En el periodo de estudio la plataforma de las IES en Venezuela, no ha sufrido modificaciones importantes en materia de nuevas instituciones en cantidades absolutas, sin embargo se observan algunos cambios en las relaciones pública - privada y mayor variación en el aumento de IES de tipo universitaria y disminución de las IES no-universitarias. Es importante señalar, que en Venezuela la creación de la Universidad Bolivariana de Venezuela y la implementación de la "Misión Sucre" han logrado reconfigurar el tejido institucional tradicional. Es así como para el desarrollo de la "municipalización" universitaria, fue necesario en primer lugar la implementación de los Ambientes Locales de Desarrollo Educativo Alternativo Socialista (ALDEAS) y sedes satelitales (expansión geográficas mayormente de las universidades experimentales, politécnicas, colegios universitarios e institutos tecnológicos) los cuales constituyen una nueva red de espacios (localizados en escuelas básicas, casas de la cultura, centros penitenciarios, entre otros espacios de instituciones oficiales o no) que son utilizados solo para la formación de profesionales. Esta nueva red de espacios alcanzó en menos de cinco años la cantidad de 1915 (ALDEAS), distribuidas en 335 municipios para el año 2009, de acuerdo con los datos ofrecidos por la Oficina de Estadística y Análisis Prospectivo del Ministerio para el Poder Popular para la Educación Universitaria (MPPEU, 2009).

Generalidades de la política de financiamiento público dirigido al sistema de educación superior

El financiamiento público de la ES Latinoamérica continúa siendo la fuente principal de los ingresos de la ES presente en la región; razón por la cual, ha sido uno de los aspectos más debatidos en las tres últimas décadas, principalmente por la influencia, impacto y diferencias que se han generado entre los índices (crecientes) de la masificación estudiantil y los índices (casi estables) de la inversión de los recursos públicos empleados para dicho sector. Son estas algunas de las dificultades, que han conllevado a los Estados latinoamericanos a considerar el financiamiento público como un elemento común en casi todas las reformas dirigidas a la ES, sin que aún se conozcan asuntos claves (cuánto cuesta la ES y cuánto debería costar; cuánto

\begin{tabular}{l|l|l|l|l|l} 
Rev. Inter. Educ. Sup. & Campinas, SP & v.3 & n.2 & p.379-400 & maio/ago. 2017 \\
\hline
\end{tabular}


se gasta efectivamente y cuánto se invierte; quiénes soportan el costo de la ES y quiénes se benefician) para el fortalecimiento de las políticas públicas del sector, en síntesis "no se ha generado mayor conocimiento en materia del financiamiento" (GARCÍA-GUADILLA, 2004).

Como ya es conocido, existen múltiples aristas al momento de estudiar el financiamiento público, además generalmente como resultado se observa que los recursos públicos son y, posiblemente siempre sean, insuficientes para el desempeño adecuado de la gestión académica de las universidades latinoamericanas; en tal sentido la presente investigación se aparta de estos postulados con el propósito de realizar un análisis de los aspectos financieros "más allá de los números e indicadores que se presentan", es decir, prestando particular atención en los mecanismos implementados para la asignación de recursos públicos e instrumentación de la política pública de financiamiento y cómo estos se corresponden en alguna medida con la política salarial de los profesores universitarios.

La política pública de financiamiento para la educación superior mexicana, ha estado condicionada y estable desde la década de los noventa. Consiste básicamente en dos modalidades bien definidas: para la primera de ellas, existe una asignación negociada de los fondos públicos ordinarios para el funcionamiento básico ${ }^{3}$ de las IES, los cuales procuran ser compensados en segundo lugar, mediante la aplicación de una estrategia de recursos extraordinarios con carácter de fondos públicos mayormente concursables entre las IES.

Para el caso de la inversión pública en materia de la ES, se observa que el gobierno mexicano ha hecho un esfuerzo público para incrementar su índice de financiamiento de la ES con relación al PIB; para el año 2000 se ubicaba en 0,57\% hasta llegar al 0,91\% en el año 2009; mientras que para el caso de la ES del sector privado se conoce que la fuente principal de ingresos está conformada por: cuotas que cobran a estudiantes, la recepción de donaciones filantrópicas y subsidios del sector público y el sector empresarial (en algunos estados existe algunas consideraciones fiscales para las empresas que contribuyan con la ES), (GIL ANTÓN, 2006; GIL ANTÓN, 2011).

La proporción de los recursos ordinarios para el funcionamiento de las IES estuvo determinada para el año 2010 en un $89,30 \%$ y, para los fondos adicionales concursables de compensación en un $10,70 \%$. Cabe señalar que no son porcentajes estándares para cada una de las IES, sencillamente porque los fondos adicionales se pueden conquistar en mayor o menor proporción, pero generalmente las IES podrán alcanzar como máximo hasta una cuarta parte del presupuesto total por la vía de los recursos adicionales concursables, estaríamos en presencia de un presupuesto ordinario del $75 \%$ y recursos adicionales logrados por $25 \%$, los

\footnotetext{
${ }^{3}$ Es preciso señalar que cerca del $95 \%$ el gasto destinado al funcionamiento de la ES latinoamericano se destina para el pago de salarios y demás gastos corrientes de funcionamiento, según el (Brunner, J.J. 2007).

\begin{tabular}{l|l|l|l|l|l} 
Rev. Inter. Educ. Sup. & Campinas, SP & v.3 & n.2 & p.379-400 & maio/ago. 2017 \\
\hline
\end{tabular}
}


cuales representan una suma de dinero importante para operar en materia de "investigación, extensión, mantenimiento, ampliación del acervo informático e inversiones para laboratorios, talleres, etc...” según lo señalado por (GIL ANTÓN, 2014, p-450).

Los fondos adicionales concursables que estuvieron disponibles para el periodo 2000-2010, fueron los siguientes: 1) El Fondo para la Modernización de la Educación Superior (FOMES); 2) El Programa de Mejoramiento del Profesorado (PROMEP); 3) El Programa de Apoyo al Desarrollo Universitario (PROADU); 4) El Fondo de Inversión para las Universidades Públicas Estatales con programas evaluados y acreditados (FIUPEA); 5) El Programa Integral de Fortalecimiento del Postgrado (PIFOP); 6) El Programa Integral de Fortalecimiento Institucional (PIFI); 7) El Fondo de Apoyo Extraordinario a las Universidades Públicas (FAEUP); 8) El Programa Integral de Fortalecimiento de la Educación Media Superior $(\text { PIFIEMS })^{4}$; 9) Fondo especial para las Universidades Públicas Estatales por abajo del costo promedio por alumno; 10) Fondo especial para el problema de las pensiones; 11) Fondo especial para el saneamiento de las finanzas institucionales (este fondo se orienta a que las IES paguen o amorticen las deudas mayormente de los servicios públicos); 12) Fondo para el reconocimiento oficial de la plantilla (este fondo se otorga para regularizar al personal que fue contratado por las IES sin el respaldo económico de los diferentes órganos del Estado). (GIL ANTÓN, 2006; GIL ANTÓN, 2011).

Para el caso venezolano, la política de financiamiento público ha sufrido modificaciones sustantivas durante el periodo de estudio, las cuales podrían contextualizarse en dos subperiodos a saber: 2000-2005 y 2005-2010; los cuales serán fundamentados principalmente por los trabajos de García-Guadilla (2006), Parra-Sandoval (2011) y Torres-Núñez (2016).

Para el primer sub-periodo 2000-2005, se conoce la presencia de un modelo de asignación de recursos presupuestarios de tipo mixto, es decir, en primer lugar, prevalece el modelo "histórico negociado" (el cual procura solo un incremento porcentual de los recursos económicos tomando en consideración las asignaciones de los años anteriores) al igual que en toda la región, solo que se procuró darle mayor sentido al considerar algunos indicadores de referencia, tales como: a) número de estudiantes y egresados; b) actividad de investigación; c) proyectos especiales y actividades de postgrados; d) número de jubilados y e) relación empleado-profesor. (GARCÍA-GUADILLA, 2006) y (TORRES-NÚÑEZ, 2016).

En segundo lugar, el modelo de financiamiento contemplaba la aplicación de indicadores operativos conocidos como "Normas CNU-1992", las cuales establecían criterios obligatorios para el financiamiento del 18,50\% condicionado de la ES, entre ellos señalamos dos grupos; los coeficientes fijos, los cuales alcanzan una proporción del 13,50\% distribuidos en: 1) coeficientes de investigación fijo $(3 \%) ; 2)$ coeficiente para postgrado $(1,5 \%) ; 3)$ coeficiente

${ }^{4}$ Este fondo, no aparece en funcionamiento para el periodo 2005-2010, (GIL ANTÓN, 2011).

\begin{tabular}{|l|l|l|l|l|l|} 
Rev. Inter. Educ. Sup. & Campinas, SP & v.3 & n.2 & p.379-400 & maio/ago. 2017 \\
\hline
\end{tabular}


para bibliotecas $(1,5 \%)$; 4) coeficientes para la extensión y cultura $(1,5 \%) ; 5)$ coeficiente para deportes $(1,5 \%) ; 6)$ coeficientes para funcionamiento y equipamiento de pregrado $(1,5 \%) ; 7)$ coeficiente para desarrollo de recursos humanos $(0,5 \%) ; 8)$ coeficientes para convenios institucionales $(0,5 \%) ; 9)^{5}$ coeficiente para crecimiento y desarrollo $\left.(0,5 \%) ; 10\right)$ coeficiente para mantenimiento $(0,75) ; 11)$ seguro de asistencia médica para los estudiantes $(0,25 \%)$. Y los coeficientes variables: coeficiente de investigación variables (5\%). (Torres-Núñez, 2016).

Para el sub-periodo 2005-2010, se observa como a partir del año 2006 el Ministerio implementa un nuevo modelo para la asignación de los recursos públicos a las IES, donde se desconocen los mecanismos y criterios aplicados, caracterizado en primer lugar, por asignar discrecionalmente los recursos en bloque por parte de los órganos del Estado para las IES; y en segundo lugar, por la implementación como nuevo elemento de financiación, que consiste en la solicitud de recursos adicionales por parte de las IES, para financiar solo una parte de aquellos gastos no cubiertos por el presupuesto ordinario, como procedimiento continúo se establece la gestión de créditos adicionales. En concreto se ha estado en presencia de administración de fondos centralizados insuficientes (que no cubren ni siquiera la compensación monetaria por la pérdida ocurrida por los altos índices de la inflación venezolana) por parte del Estado para dejar constancia de la mayor crisis presupuestaria y salarial que presenciaron las IES venezolanas para la primera década del siglo XXI, a pesar de la presencia de los mayores ingresos públicos que obtuvo la nación, de acuerdo a los indicadores macroeconómicos que corresponden a este sub-periodo. (Torres-Núñez, 2016).

Finalmente en este punto, se observa la correspondencia entre las políticas de asignación de recursos públicos y el establecimiento de los salarios de los académicos; en el caso mexicano, la asignación inicial del presupuesto para las IES solo cubre gastos corrientes y salarios "base" de los profesores, por tal razón las IES y los académicos que requieran incrementar sus recursos para el desarrollo de la gestión académica y otros requerimientos deben acudir a los diferentes programas de financiamiento para tales fines. Mientras que en el caso venezolano, la homologación de los salarios, ha sucumbido en relación a los términos iniciales en la cláusula 13 de las Normas de Homologación, la cual prevé que los salarios de los profesores universitarios deberán revisarse cada dos años y se tomará como referencia los IPC de los años en cuestión. La primera violación de esta cláusula fue registrada para el año 1985, a solo tres años de su entrada en vigencia, en lo sucesivo su cumplimiento ha sido a medias en algunas ocasiones más que en otras. Al parecer, las asignaciones presupuestarias para las IES, han corrido la misma suerte, se dejó atrás los criterios o normas CNU, toda vez que los recursos públicos se han visto en merma por las razones ya señaladas en el aparatado anterior.

\footnotetext{
${ }^{5}$ Los numerales 9, 10, 11 y 12 fueron nuevos fondos creados para el periodo 2005-2010, (Gil Antón, 2011). 


\section{LOS PROFESORES UNIVERSITARIOS}

Son actores fundamentales para el desarrollo académico de los sistemas nacionales de educación superior. La alta tasa de masificación de la ES en la región latinoamericana ha logrado impactos fundamentales que podría estar afectando en desarrollo adecuado de la profesión académica, entre ellos podríamos mencionar la orientación mayormente hacia la actividad de docencia (en menoscabo de la actividad científica) y la presencia de bajas remuneraciones. Es una variable compleja para el estudio científico, carece de trabajos empíricos en la mayoría de los países y además de la falta de publicación de datos oficiales que permitan explicar las condiciones fundamentales y la situación de los profesores para cualquier periodo en estudio (García-Guadilla, 1998).

Tabla 4: Evolución del número de profesores universitarios en México.

\begin{tabular}{lccc}
\hline Años & Pública & Privada & Total \\
\hline$* 2000-2001$ & s.d & s.d & 208692 \\
$* 2001-2002$ & s.d & s.d & 219804 \\
$* 2002-2003$ & s.d & s.d & $231558^{(1)}$ \\
$* 2003-2004$ & s.d & s.d & 241236 \\
$* 2004-2005$ & s.d & s.d & 251740 \\
$* * 2005-2006$ & 157226 & 98070 & 255296 \\
$* * 2006-2007$ & 160875 & 100070 & 260945 \\
$* * 2007-2008$ & 170939 & 108947 & 279886 \\
$* * 2008-2009$ & 170805 & 113013 & 283818 \\
$* * 2009-2010$ & 157226 & 98070 & 255296 \\
\hline
\end{tabular}

\section{Fuente:}

* Los datos fueron obtenidos de las publicaciones estadísticas de la Secretaria de Educación Pública.

** Los datos fueron tomados del Informe Nacional de la Educación Superior en Iberoamérica. Capitulo México (Gil Antón y Pérez, 2011).

s.d: sin datos; los estudios antes señalados, muestran la información para este periodo en términos globales.

(1) Solo para este periodo, se observó la estratificación de la dedicación del tiempo al trabajo académico, dato valioso para la investigación.

En relación a los datos señalados en el tabla 4 "Evolución del número de profesores universitarios en México" se observa el incremento de cerca de 50.000 nuevas plazas docentes (varias plazas académicas pueden ser ocupadas por el mismo académico, por ello se diferencian el número de plazas del número de personas) en el periodo en estudio. Un elemento fundamental lo constituyen los datos señalados para el periodo (2002-2003) del cual también se conoció que la planta profesoral estaba dividida en dedicación en la siguiente proporción: $22.64 \%$ de profesores a tiempo completo (TC); 6,11\% de profesores a medio tiempo (MT) y 54,42\% de profesores contratados por horas. Aun cuando se desconoce el escalafón que ellos ostentan, es sencillo inferir sobre la fuerte influencia (de carácter 
docentista) que está ejerciendo la mayoritaria proporción de profesores por horas sobre el sistema educativo mexicano, de tal forma que es preciso señalar lo planteado por Gil Antón y otros, (2012), que la masificación no ha logrado impactar seriamente sobre el número de cargos a TC, los cuales se han mantenido casi invariables desde 1994 (30\%) a un (31\%) para el año 2005; generando en este sentido ciertas condiciones de estabilidad en las políticas dirigidas a la profesión académica, la cual a su vez se encuentra influenciada por diversas políticas sectoriales, principalmente por la política de financiamiento, la cual la conduce a múltiples programas de compensación salarial.

Para la caracterización general de los académicos mexicanos, fue necesario completar la información de los datos contenidos principalmente en los trabajos de Gil Antón (2011); Gil Antón y otros (2009); Galaz Fontes (2008) y otros trabajos académicos en esta área, de la cual se pueden destacar información pertinente para la presente investigación. El grupo de los académicos mexicanos históricamente ha estado mayoritariamente conformado por hombres, los cuales aún para el año 2007 representan el 64\% del total de la población académica; la edad promedio de los académicos mexicanos es de 49,50 años (una planta académica madura); además se ha observado en el periodo de estudio la incorporación tardía de nuevos académicos, situados en una edad promedio de 37,50 años, pero con estudios de posgrados ya culminados; la cantidad de TC se encuentran en su mayoría en las universidades públicas, 87\% para el año 2008; el tiempo empleado para la actividad académica está promediado en 43,50 horas semanales, con mayor énfasis en la actividad de docencia y apoyo a la actividad de docencia (22,50 horas).

Tabla 5: Evolución del número de profesores universitarios en Venezuela.

\begin{tabular}{lllllllllll}
\hline \multicolumn{9}{c}{ Número de Académicos } & \multicolumn{9}{c}{ Discriminación en los escalafones académicas } \\
& Pública & Privada & Total & Instructores & Asistentes & Agregados & Asociados & Titulares & Horas** \\
\hline 2000 & s.d & s.d & $49745^{*}$ & s.d & s.d & s.d & s.d & s.d & s.d \\
2001 & s.d & s.d & s.d & s.d & s.d & s.d & s.d & s.d & s.d \\
2002 & s.d & s.d & s.d & s.d & s.d & s.d & s.d & s.d & s.d \\
2003 & s.d & s.d & s.d & s.d & s.d & s.d & s.d & s.d & s.d \\
2004 & s.d & s.d & s.d & s.d & s.d & s.d & s.d & s.d & s.d \\
2005 & 49709 & 16719 & 66428 & s.d & s.d & s.d & s.d & s.d & s.d \\
2006 & 44378 & 20205 & 64583 & s.d & s.d & s.d & s.d & s.d & s.d \\
2007 & 73840 & 29584 & 103424 & 8659 & 6813 & 5185 & 3657 & 2388 & 47138 \\
2008 & 68868 & 30641 & 99509 & 8836 & 6121 & 4815 & 4256 & 3911 & 40929 \\
2009 & 66592 & 29609 & 96201 & 9995 & 6239 & 3772 & 5099 & 2205 & 39282
\end{tabular}

Fuente: Los datos fueron tomados de los dos Informes Nacionales de la Educación Superior en Iberoamérica. Capitulo Venezuela (Parra-Sandoval 2011).

s.d: sin datos; en los estudios antes señalados, no existe información oficial publicada al respecto, ni trabajos empíricos que contengan la información requerida.

*Estimación de los profesores para el año 2000, hecha por Víctor Morles (2003).

** Reconstrucción de los datos faltantes (con excepción de los académicos del sector privado), se estiman que son los que se encuentran en las dedicaciones de: Medio Tiempo (MT) y horas menores a 7 horas. 
La población de profesores universitarios para el caso de Venezuela, pasó el año 2000 de 49745 profesores a 96.201 profesores para el año 2009, cifra que representa un incremento de casi el doble del número las plazas académicas para ubicarse porcentualmente en 93,38\%. La distinción académica entre los diversos escalafones de la carrera académica, nos indican que tenemos una planta académica completamente novel y/o de poca formación académica, al considerar que los dos últimos escalafones de la profesión académica (titulares y asociados) alcanzan en conjunto solo el 10,97\% de la totalidad de los profesores universitarios del sector público; lo que es peor, los datos nos indican que el 58,99\% de los profesores venezolanos son Medio Tiempo (media jornada diaria) y podrían ser profesores contratados por horas (menores a 7 horas semanales de trabajo), es decir, son profesionales que ocasionalmente se dedican a "dictar algunas horas de clase a la semana", desde luego sin tiempo para atender la actividad científica que le es propia a esta profesión en la universidad pública venezolana, la cual solo está siendo medianamente atendida por el $41,01 \%$ de profesores a tiempo completo y dedicación exclusiva; a diferencia de México, en Venezuela la política de universalización mediante la estrategia de municipalización en "nuevos espacios" está afectando seriamente la formación y desarrollo científicos de la gran cantidad de profesores noveles.

Lamentablemente, la inexistencia de datos estadísticos públicos de los órganos del Estado y las IES, hacen complejo continuar profundizando en el análisis e interpretación de los académicos venezolanos en dicho periodo; no se puede señalar el tiempo efectivo dedicado a la actividad académica, la edad promedio, la proporción del género empleado en el sector universitario y, cuál es la tasa de jubilados que aún está prestando sus servicios dentro de los recintos universitarios, a pesar de ya haber culminado su tiempo de servicio.

\section{LA POLítica SALARIAL DE LOS ACADÉMICOS}

El estudio de la política salarial académica, demanda del conocimiento previo de las consideraciones filosóficas establecidas por las Naciones Unidas y la Organización Internacional del Trabajo (OIT), toda vez que procura estricto cumplimiento de la Declaración Universal de los Derechos Humanos, la cual establece en su artículo 23, 3) el derecho que tendrá toda persona que trabaja a recibir "una remuneración equitativa y satisfactoria, que le asegure, así como a su familia, una existencia conforme a la dignidad humana y que será completada, en caso necesario, por cualesquiera otros medios de protección social” (ONU, 1948).

Ahora bien, de acuerdo con la Conferencia Internacional del Trabajo número 79 de fecha 1992, se ratifica que la concepción de "salario" la cual está referida a todo tipo de remuneración o ganancia, que independientemente del método del cálculo, siempre se pueda evaluar en términos de dinero, fijados por un empleador mediante contrato (verbal o escrito) por el trabajo que el trabajador deberá realizar, siempre que esté conforme a una legislación 
nacional. Asimismo, se entenderá por remuneración, todo el salario ordinario, básico o mínimo y cualquier otro emolumento pagado en dinero o especies por el empleador, de forma directa o indirecta al trabajador en el cumplimiento del concepto del empleo.

Para conocer la concepción salarial "conforme a la legislación nacional" hemos decido la revisión de las constituciones vigentes para los dos Estados objetos de la investigación, en el entendido que toda ley, reglamento o norma que se promulga en material salarial debería tener correspondencia al texto constitucional. En el caso de la Constitución Política de los Estados Unidos Mexicanos (1917), señala en su artículo 123, que los salarios mínimos se estructuran en: generales y profesionales, los cuales deberán "ser suficientes para satisfacer las necesidades normales de un jefe de familia, en el orden material, social y cultural ...". Mientras que para el caso venezolano la Constitución de República Bolivariana de Venezuela (1999) señala en su artículo 91, que el trabajador tendrá "derecho a un salario suficiente que le permita vivir con dignidad y cubrir para sí y su familia las necesidades básicas materiales, sociales e intelectuales...". En ambos casos, se observa el cumplimiento de las disposiciones de la ONU y la OIT en los cuerpos constitucionales, toda vez que se refleja tácitamente que el salario deberá propiciar las condiciones de equidad, dignidad al ser humano y además debe ser satisfactorio no solo para el trabajador, sino para su núcleo familiar.

A pesar de que ambos países presentan similitudes filosóficas y legales entorno a la concepción del salario, los salarios de los académicos mexicanos y venezolanos compartieron lógicas conceptuales y estructurales similares hasta hace tres décadas; la génesis de la diferenciación conceptual del salario académico ha sido producto de la crisis latinoamericana en materia fiscal y de funcionamiento del aparato público, mejor conocida como "crisis del Estado intervencionista, paternalista y centralista", que se desató en el año de 1982, una vez que México se declaró en estado de Mora internacional (LORA, E. 2007).

Para el año 1982, los académicos mexicanos presencian la mayor crisis salarial, registrando una pérdida del poder adquisitivo que se ubicó en un $60 \%$. Allí se abandona el método de los "incrementos generales de salarios" (salarios homologados hoy en Venezuela) el cual consistía en la recuperación de los ingresos salariales perdidos entre los acuerdos contractuales de la IES y los sindicatos; pasando, a un nuevo mecanismo conocido como "estructura de incentivos" (salarios heterologados) el cual consiste en primer lugar en la determinación de un salario mínimo "base", determinado por las IES (en algunos casos solo abarca el 30\% de los ingresos mensuales que perciben los académicos) y, en segundo lugar, la posibilidad de que cada académico pueda complementar sus bajos ingresos mediante la participación en uno o varios programas de estímulos, becas o premios por mérito (en los mejores casos llegan a abarcar hasta el 70\% de los ingresos salariales mensuales que perciben los académicos); para instaurar una estructura salarial académica diversa entre las IES y caracterizada por la multiplicidad de criterios salariales base y complementarios. (GIL ANTÓN, 2014). 
En el caso de los salarios de los académicos venezolanos, producto de la crisis salarial de los académicos, el acuerdo entre el Estado, las IES y los representantes gremiales fue la instauración de un mecanismo que lograra la recuperación bianual del salario tomando a las tasas de inflación (IPC) publicadas por el BCV; es así como en agosto del año 1982 se firma el primer acuerdo contractual conocido como las Normas de Homologación (NH); las NH no solo indexan el salario al IPC sino que además establecieron la unidad salarial de todos los profesores universitarios en Venezuela, tomando en consideración solo las diferencias en escalafón de la carrera académica y el tiempo de dedicación laboral; posteriormente algunas IES iniciaron en el marco de su autonomía, algunas consideraciones gremiales adicionales (Actas Convenios) las cuales, también han sido homologadas en la mayoría de las IES llevando la misma uniformidad salarial y demás beneficios socio-académicos a los profesores venezolanos; para instaurar una estructura salarial académica uniforme entre las IES y caracterizada por la unidad de criterios salariales (PARRA-SANDOVAL, 2011).

En términos de la contratación, los académicos mexicanos pueden experimentar básicamente dos vías de ingreso, el asalariado para el caso de los profesores que trabajan en la IES del sector público, ya sea en cargos permanentes o contratados (independientemente de las modalidades de tiempo de dedición al trabajo) y el profesional contratado bajo la modalidad de honorarios profesionales, la cual es una modalidad mayormente utilizada para las IES del sector privado (MALDONADO, 2011).

La contratación de los académicos venezolanos es de dos tipos, para las IES del sector público: en primer lugar mediante el concurso de oposición, el cual le garantiza la permanencia fija en el cargo y el derecho a realizar su prosecución en la carrera académica y en segundo lugar, mediante un concurso de credenciales o revisión de documentos para la contratación por tiempo determinado y mayormente de carácter renovable, hasta realizar la modalidad señalada anteriormente. En el caso de las IES del sector privado, el mecanismo mayormente utilizado es la contratación de personal de acuerdo con los criterios establecidos por la misma IES, el cual no garantiza la estabilidad laboral para los profesores (PARRASANDOVAL, 2011).

El estudio de comparación salarial de los académicos mexicanos y venezolanos, procura superar dos limitaciones fundamentales. La primera, la casi inexistente información de los salarios académicos mexicanos, se debe comprender que al ser las IES quienes generan sus propias políticas en materia de contratación y determinación salarial de los académicos, determina un diverso panorama "salarial" supremamente estratificado y heterogéneo en todo el país, lo cual dificulta su estandarización adecuada. En segundo lugar, la presencia de un control cambiario en la economía venezolana, situación que desdibuja el valor monetario de los salarios académicos, cuando son expresados en moneda internacional para facilitar su comparación. Sin embargo, para avanzar en los fines de la presente investigación se tomaron en primer lugar los datos de los salarios de la carrera académica de ambos países para el año 
2009 y se transformaron de Dólar Americano, y se procedió aplicar el cálculo para una unidad de comparación denominada salario equivalente SE, quedando la información detallada en el tabla número 6 "Salarios de los profesores mexicanos y venezolanos, expresados en unidades de salarios mínimos respectivos":

Tabla 6: Salarios de los profesores mexicanos y venezolanos, expresados en unidades de salarios mínimos respectivos.

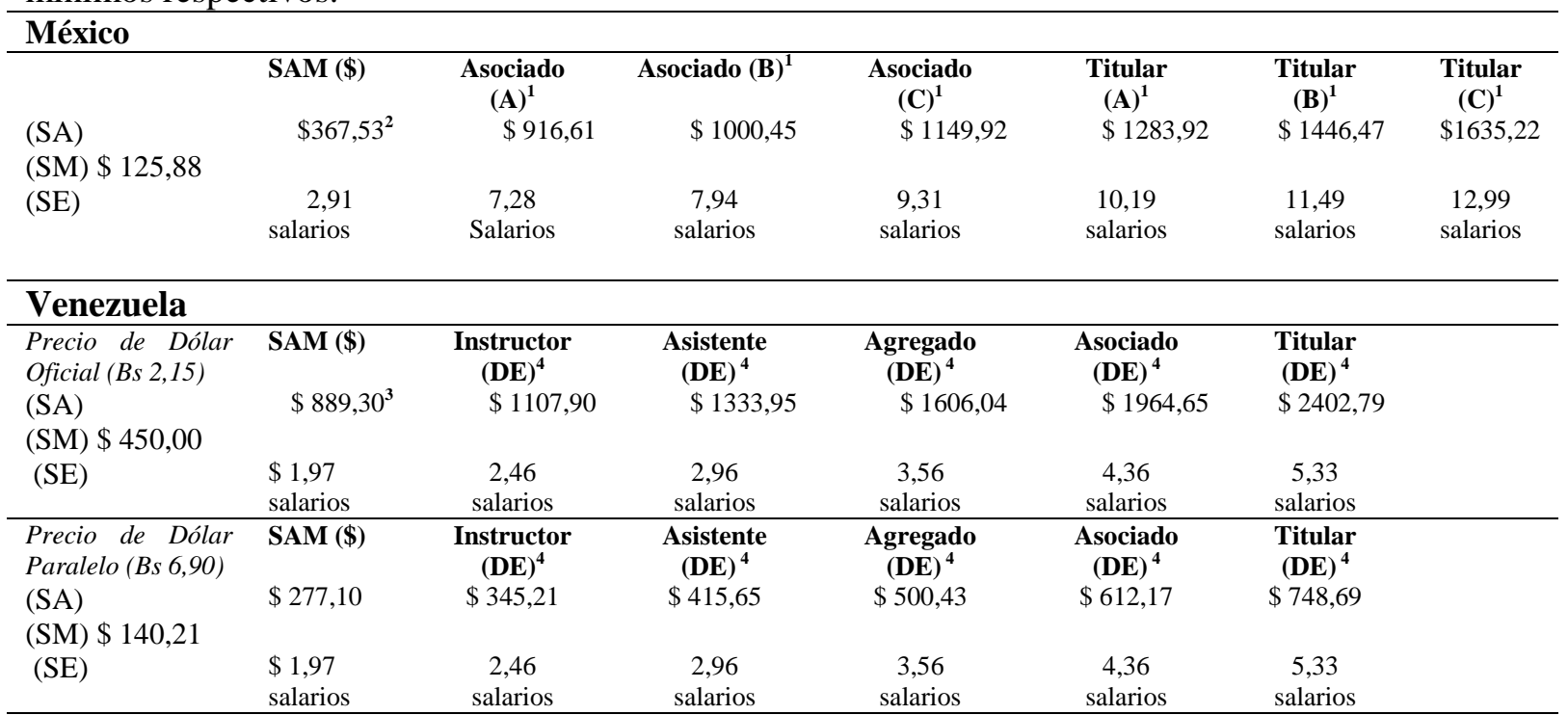

\section{Fuente:}

${ }^{1}$ Los datos salariales de las seis categorías e incluso su denominación. No se lograron obtener los datos de los salarios bases, en tal sentido los allí presentados incluyen los otros emolumentos (MALDONADO, A. 2011).

${ }^{2}$ El salario más bajo cancelado en las IES públicas en México para el año 2009, no se conoció ni su dedicación ni escalafón (MALDONADO, A. 2011)

${ }^{3}$ Salario del instructor tiempo completo (TC) según el decreto de aumento salarial del Ministerio para el año 2008.

${ }^{4}$ Se toman en consideración solo los salarios de los profesores a dedicación exclusiva (DE) quienes son los que mayores salarios devengan en Venezuela.

SA= Salario Académico (año 2009)

SM=Salario Mínimo (año 2009)

$\mathrm{SE}=$ Salario equivalente, resulta de transformar el salario académico entre el salario mínimo nacional.

SAM=Salario Académico Mínimo (año 2009)

Tasa de Cambio para el 31 de diciembre 2009 Venezuela México=13,06 (BM)

Tasa de Cambio para el 31 de diciembre 2009 Venezuela= 2,15 (BCV).

Tasa de Cambio para el 31 de diciembre 2009 Venezuela $=6,90$. 


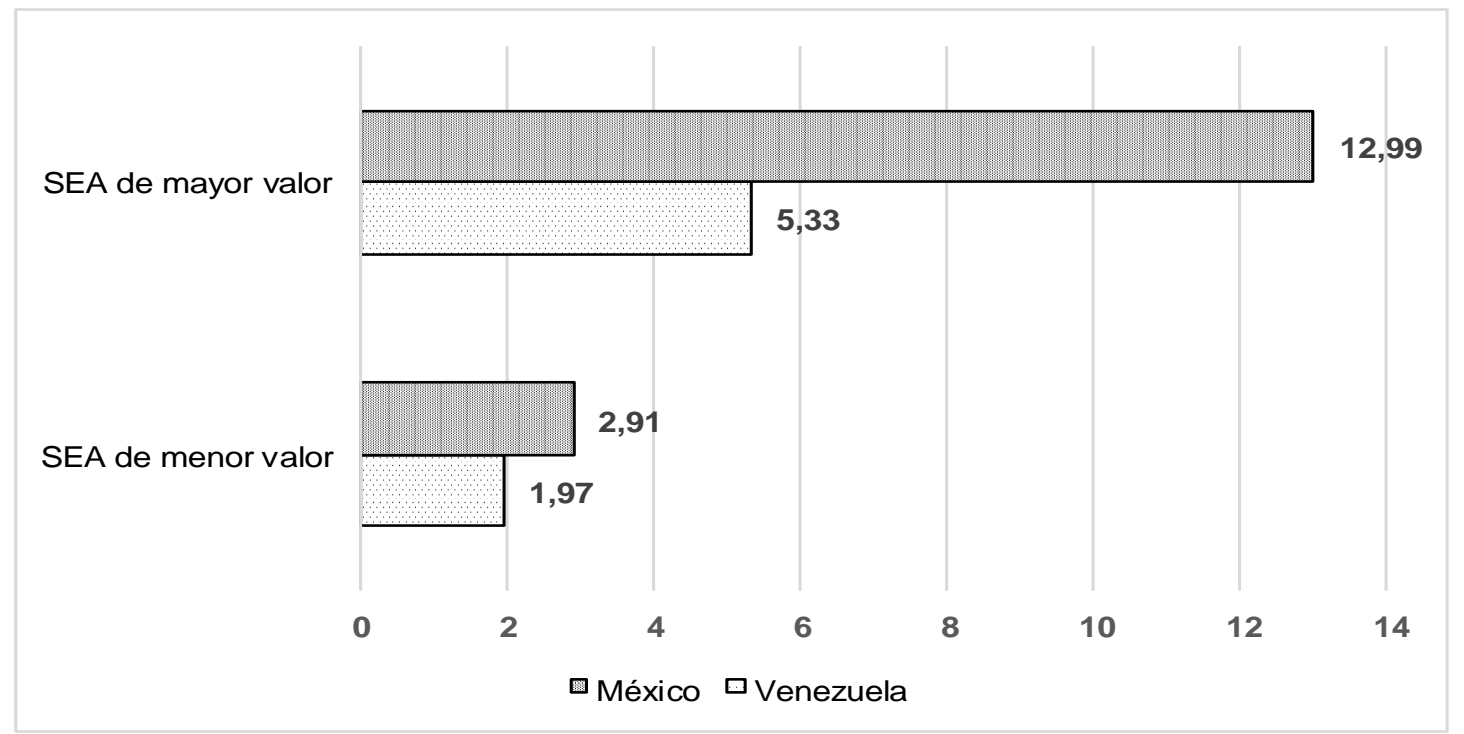

Grafico 3: Relación de los salarios equivalentes académicos

Los salarios equivalentes académicos (SEA) de los profesores universitarios expresados en salarios mínimos procuran normalizar los datos de los países en estudio. Se observa para el caso venezolano, cómo los salarios son realmente mucho más bajos que los salarios de sus pares mexicanos; el profesor venezolano con más de 15 años de carrera académica y/o trabajos de investigación publicados (en cumplimiento de la normativa nacional para acceder al máximo grado de la carrera académica) alcanza como máximo 5,33 SEA; es decir, solo 2,42 SM más que el profesor novel con menos credenciales académicas que devenga la menor cantidad de SEA en las IES públicas mexicanas (MALDONADO, 2011).

Queda en evidencia, que el salario base de los profesores mexicanos también es bajo. Para que un profesor mexicano pueda devengar 4,37 SAE adicionales y llegar a ubicarse en el caso de los profesores asociados (A), requiere acudir obligatoriamente a la estructura de incentivos económicos; pero en definitiva se debe entender, que este ingreso -vía incentivos- solo resuelve el problema económico inmediato del académico, al ser considerado por las IES mexicanas como un ingreso de tipo "no salarial" que no tendrá incidencia en la previsión social del trabajador, principalmente en el fondo para jubilarse.

En este punto es preciso señalar, que no todos los profesores universitarios en México ganan 12,99 salarios mínimos; recordemos que solo 22,64\% son los que se dedican exclusivamente a la actividad académica, pero además cumplen con un promedio de 22,50 horas semanales dedicadas solo a la actividad de docencia, es claro que el tiempo empleado para la investigación y demás trabajos propios del concurso y evaluación para el reconocimiento salarial adicional por la vía de los incentivos, les queda cuesta arriba. 


\section{CONSIDERACIONES FINALES}

En el presente estudio se evidencia como la crisis económica ocurrida en México y Venezuela, abrió la posibilidad para construir e implementar una política salarial para los académicos de ambos países, y que ha tenido una importante influencia en los sistemas educación superior en el periodo de estudio 2000-2010.

La política de las asignaciones adicionales al salario básico mediante la "estructura de incentivos", implementó una política de heterologación de los salarios de los académicos mexicanos que ha permanecido estable en las tres últimas décadas, a pesar de la violación expresa a su texto constitucional. Esta política de salario mexicana, es el resultado de la misma política de financiamiento del sector universitario; es decir, sin la estructura de incentivos económicos es imposible proveerse de mayores recursos para el beneficio de la gestión académica de las universidades públicas y la complementación salarial de los académicos principalmente.

Está política mexicana ha logrado con éxito, el reconocimiento científico de varias universidades públicas y centros de investigación, además del fortalecimiento científico de su plantilla académica, todo ello a pesar de no lograr incrementar su tasa de profesores dedicados a TC a la actividad académica, situación que ha permanecido casi invariable en los últimos 15 años; ahora bien, la política salarial ha generado sus amenazas a la gestión académica, principalmente porque su planta académica envejece y quienes han ingresado a la profesión académica en el periodo de estudio, ya promedian edad avanzada (37,5 años); es decir que estamos en presencia de lo que varios autores han denominado los efectos "indeseados" del sistema mayormente basado en incentivos no salariales.

Sin lugar a dudas, la política salarial mexicana de estructura de incentivos debe ser modificada para evitar que la misma continúe afectando el desarrollo de las generaciones futuras y la protección social de los académicos adultos, permitir en primer lugar que los profesores jubilados puedan participar de ella en alguna medida y en segundo lugar, se debe modificar algunos criterios para considerar estos ingresos adicionales como definición legal del "salario" real de los académicos y sus implicaciones en las demás consideraciones propias de la previsión social del trabajador.

En relación a la política salarial emprendida por Venezuela, se mantuvo la senda de la contratación laboral $(\mathrm{NH})$, la cual pretendía el acuerdo entre el Estado, las IES y los representantes gremiales mediante la aplicación de un mecanismo que procuraba la actualización del salario bianualmente en referencia a los IPC de los referidos años. Se observó el incumplimiento continúo en la indexación del salario por parte del Estado, lo cual ha conllevado a una situación del deterioro estrepitoso ya acumulado por años para los 
salarios académicos; además está política de "salarios homologados" no reconoce las diferencias de la productividad científica, situación que no solo genera una "desmotivación del académico" que en el tiempo podría ser traducida como la causa del abandono en la actividad académica o la movilidad a otras latitudes en búsqueda de otros centros científicos con mejores oportunidades de desarrollo científico y personal, todo esto en prejuicio de la gestión académicas que deben desarrollar las universidades venezolanas (GARCÍAGUADILLA, 1998).

El ingreso masivo de nuevos profesores universitarios para la atención de la matrícula en la década de estudio, debería haber estado acompañado de una nueva política salarial que rompiera con la homologación "exclusiva" de los salarios académicos; Venezuela debe cambiar la relación de profesores MT y por horas a profesores TC y DE, además de garantizar las oportunidades de formación inicial de los académicos noveles e incluso hacer atractiva la profesión académica para otro tipo de profesionales destacados en otras áreas de la actividad económica del país, si en realidad se procura incrementar el desarrollo científico de las universidades venezolanas.

Finalmente al realizar el estudio comparativo de los salarios académicos a unidades equivalentes para los dos países, encontramos que la estructura mexicana de salarios académicos de tipo heterologados demuestra mayor beneficio económico que la política venezolana de salarios de tipo homologados, toda vez que se conoce que la política salarial de tipo homologada ha sido irrespetada por el Estado Venezolano desde hace más de tres décadas, impidiendo que los profesores universitarios y su núcleo familiar gocen de una verdadera vida digna como lo señala su texto constitucional. Ahora bien, es importante aclarar que los beneficios económicos que reporta la política de incentivos para los académicos mexicanos, son de nulo impacto en su previsión social, es decir, son beneficios económicos de carácter inmediato para la sobrevivencia del académico en ese momento y no le garantizan una pensión digna para el disfrute de su vejez, también en contrasentido de la fundamentación filosófica de salario que bien señala su norma constitucional.

\section{REFERENCIAS}

BRUNNER José Joaquín; FERRADA HURTADO Rocio. Educación superior en

Iberoamérica: informe 2011. Santiago de Chile, 2011. Disponible en: http://www.cinda.cl/download/informe_educacion_superior_iberoamericana_2011.pdf [consulta: mayo, 25, 2012].

BRUNNER José Joaquín; VILLALOBOS Cristóbal. Políticas de educación superior en Iberoamérica 2009-2013. Ediciones Universidad Diego Portales. Santiago de Chile, 2014. Disponible en: http://www.brunner.cl/?p=10365 [Consulta: 2014, julio, 25]. 
BRUNNER José Joaquín. Educación superior en Iberoamérica: informe 2007. Santiago de Chile, 2007. Disponible en:

http://www.cinda.cl/download/informe_educacion_superior_iberoamericana_2007.pdf [consulta: junio, 09, 2009].

ESTADOS UNIDOS DE MEXICO. Constitución Política de los Estados Unidos Mexicanos. México, 2017.

GARCÍA GUADILLA, Carmen. Informe de Venezuela. Proyecto CINDA: Educación superior en Iberoamérica 2006. Caracas, 2006. Disponible en:

www.carmengarciaguadilla.com/articulos/2006_EducSup_en_Iberoamerica.pdf [Consulta: 2015, Agosto, 19].

GARCÍA GUADILLA, Carmen. Situación y principales dinámicas de transformación de la educación superior en américa latina. CRESALC-UNESCO. Caracas, (1998).

GARCÍA GUADILLA, Carmen. Tensiones y transiciones. Educación superior latinoamericana en los albores del tercer milenio. CENDES-UCV. Caracas, 2004.

GALAZ FONTES, Jesús F. Los académicos mexicanos a principios del siglo XXI: Una primera exploración sobre quiénes son y cómo perciben su trabajo, sus instituciones y algunas políticas públicas. Tabasco, 2008.

GIL ANTÓN, Manuel y PÉREZ GARCÍA, María J. Proyecto informe de la educación superior Iberoamericana: México. México DF, 2006. Disponible en: http://www.cinda.cl/wp-content/uploads/2014/07/2007-Informe-M\%C3\%A9xico.pdf [Consulta: 2015, Julio, 25].

GIL ANTÓN, Manuel y PÉREZ GARCÍA, María J. Proyecto informe de la educación superior Iberoamericana: México. México DF, 2011. Disponible en:

http://www.cinda.cl/wp-content/uploads/2014/07/2011-Informe-M\%C3\%A9xico.pdf

[Consulta: 2015, Julio, 25].

GIL ANTÓN, Manuel. La educación superior en México: los costos de las monedas. En Brunner, Villalobos (2014) Políticas de la educación superior en Iberoamérica, 2009. 2013. Ediciones Universidad Diego Portales. Santiago de Chile 2014.

GIL ANTÓN, Manuel; GALAZ FONTEZ, Jesús. Francisco; PADILLA GONZÁLEZ, Laura; SEVILLA GARCÍA, Juan; ARCOS VEGA, José; MARTÍNEZ SNACK, Jorge. et al. La profesión académica en México: Continuidad, cambio y renovación. En Fernández Lamarra (2012) El futuro de la profesión académica: Desafíos para los países emergentes. Buenos Aires, Argentina 2009.

LORAS Eduardo. La reforma del Estado en América Latina: una revolución silenciosa. En LORAS Eduardo (2010) El estado de las reformas del Estado en América Latina. Banco Mundial, Ediciones Mayol. Washington DC, 2007. 
MALDONADO Alma. Salario de académicos de instituciones de educación superior en México comparado con 30 países. Ponencia presentada en XI congreso nacional de investigación educativa. México 2011. Disponible en: http://www.comie.org.mx/congreso/memoriaelectronica/v11/docs/area_16/2395.pdf [Consulta: 2015, julio, 30].

MINISTERIO PARA EL PODER POPULAR DE LA EDUCACIÓN UNIVERSITARIA. La revolución bolivariana en la educación superior: 10 años de logros. Caracas, 2009. Disponible en: <http://venezuela-us.org/es/wp-content/uploads/2009/09/10-anos-de-logrosjunio-2009.pdf $>$.

MINISTERIO PARA EL PODER POPULAR DE LA EDUCACIÓN UNIVERSITARIA. Memoria y cuenta. Tomo I. Caracas, 2008.

MINISTERIO PARA EL PODER POPULAR DE LA EDUCACIÓN UNIVERSITARIA. Memoria y cuenta. Tomo I. Caracas, 2010.

ORGAIZACIÓN DE ESTADOS IBEROAMERIANOS. Metas educativas 2021 Documento final. Organización de Estados Iberoamericanos para la Educación, la Ciencia y la Cultura. Disponible en: http://www.oei.es/metas2021.pdf [Consulta: 2014, julio, 25] 2011.

ORGANIZACIÓN DE LAS NACIONES UNIDAS. Declaración universal de los derechos humanos. Paris, 1948. Disponible en: http://www.un.org/es/documents/udhr/ PARRA SANDOVAL, María Cristina. La educación superior en Iberoamérica 2011. La educación superior en Venezuela 2005-2009. Maracaibo, 2011. Disponible en: http://www.cinda.cl/wp-content/uploads/2014/07/2011-Informe-Venezuela.pdf [Consulta: 2015, julio, 25].

\section{REPÚBLICA BOLIVARIANA DE VENEZUELA. Constitución de la República}

Bolivariana de Venezuela. Caracas, 1999.

TORRES NÚÑEZ, Luis E. Criterios a considerar para la financiación de la educación superior en Venezuela. Revista Internacional de Educação Superior Vol. 2, Núm. 3 (2016): set./dez. - Temática: Avaliação e Regulação da Educação Disponible en: http://ojs.fe.unicamp.br/ged/RIESup/article/view/7664 [Consulta: 2016, diciembre, 27].

\section{${ }^{\mathrm{i}}$ Sobre o autor}

\section{Luis E. Torres-Núñez.}

E-mail: torresl@unesur.edu.ve / ORCID: http://orcid.org/0000-0002-2005-9101

Universidad Nacional Experimental Sur del Lago - Venezuela

Doctorado Latinoamericano em Educación pela Universidad Nacional Experimental Sur del Lago [UPEL]. 